\title{
Effectiveness of 18F-fluoro-deoxyglucose Positron Emission Tomography/Computed Tomography and Magnetic Resonance Imaging to Detect Iliopsoas Tendonitis after Total Hip Arthroplasty: A Case Report
}

\author{
Makoto Kimura, MD, Nobuhiro Kaku, MD, PhD, Hiroaki Tagomori, MD, Hiroshi Tsumura, MD, PhD \\ Department of Orthopaedic Surgery, Faculty of Medicine, Oita University, Yufu City, Japan
}

Disorders involving artificial joints are difficult to evaluate due to metal artifacts hindering plain computed tomography (CT) or magnetic resonance imaging (MRI). In the current case study $18 \mathrm{~F}$-fluoro-deoxyglucose positron emission tomography (18F-FDG-PET)/CT and MRI were used to confirm iliopsoas tendonitis within the metal artifact area following total hip arthroplasty (THA). The patient was a 61-year-old woman who developed hip pain on flexion of the ipsilateral hip after THA. Imaging studies were performed to evaluate for iliopsoas tendonitis due to impingement with the cup. Assessment of the iliopsoas muscle near the artificial joint was difficult due to the metal artifact on plain CT and MRI. Imaging using 18F-FDG-PET/CT and 18F-FDGPET/MRI showed uptake along the iliopsoas muscle. Therefore, revision was performed to resolve iliopsoas tendon impingement, and the preoperative pain resolved. The result of the current case study suggest 18F-FDGPET/CT or 18F-FDG-PET/MRI will be useful to detect iliopsoas tendonitis within metal artifact areas after THA.

Key Words: Positron-emission tomography, Tendinopathy, Magnetic resonance imaging, Hip, Arthropathy

Submitted: July 31, 2020 1st revision: September 24, 2020

Final acceptance: October 12, 2020

Address reprint request to

Nobuhiro Kaku, MD, PhD

(https://orcid.org/0000-0002-4041-1870)

Department of Orthopaedic Surgery, Faculty of Medicine, Oita

University, 1-1 Idaigaoka Hasama-machi, Yufu City, Oita 879-

5593, Japan

TEL: +81-97-586-5872 FAX: +81-97-586-6647

E-mail: nobuhirodoita-u.ac.jp

This is an Open Access article distributed under the terms of the Creative Commons Attribution Non-Commercial License (http://creativecommons. org/licenses/by-nc/4.0) which permits unrestricted non-commercial use, distribution, and reproduction in any medium, provided the original work is properly cited
Following total hip arthroplasty (THA), the prevalence of pain ranges from $0.4-18.3 \%{ }^{1}$. Often the cause of pain is unknown, even after performing various diagnostic imaging procedures, and is therefore referred to as pain of unknown etiology ${ }^{2,3)}$. One source of pain after THA is iliopsoas tendonitis due to impingement with the cup, with a reported incidence of approximately $4 \%$. . THA with an inappropriate size and/or installation angle of the cup for anterior acetabular dysplasia, which often has less bony cover, is associated with a higher incidence of iliopsoas tendonitis ${ }^{5-7)}$.

Inflammation of the muscle or tendon with edema or bursitis can be easily detected by plain computed tomography (CT) or magnetic resonance imaging (MRI) as plain CT and MRI are useful for muscle and soft tissue imaging. 


\section{Hip \& Pelvis}

Hip Pelvis 32(4): 223-229, 2020

Though it is possible that inflammation of the muscle and tendon away from the artificial joint can be visualized by plain CT or MRI, detection becomes more difficult in the presence of metal artifact when it is close to the artificial joint. Therefore, imaging of iliopsoas tendonitis near the cup is challenging with plain CT or MRI.

Recently, reports have suggested 18F-fluoro-deoxyglucose positron emission tomography (18F-FDG-PET) is useful for evaluation of inflammatory diseases such as infections and neoplasms ${ }^{8}$. However, there are no reports on the use of 18F-FDG-PET in patients with suspected iliopsoas tendonitis. For the present case report, we hypothesized that 18F-FDG-PET/CT and 18F-FDG-PET/MRI could aid in detecting iliopsoas tendonitis due to impingement with the cup, even if the lesion was close to the artificial joint. Therefore, we attempted to use 18F-FDG-PET/CT and 18FFDG-PET/MRI to evaluate a patient who suffered from groin pain due to iliopsoas muscle impingement.

\section{CASE REPORT}

This case report was approved by the Institutional Review Board (IRB) at the Oita University (IRB No. 1396; Date: April 16, 2018), and informed consent was obtained from the patient.

The patient was a 61-year-old woman who worked in an agriculture. She was diagnosed with osteoarthritis associated with acetabular dysplasia at 53 years of age and underwent left femoral curved intertrochanteric varus osteotomy with a right THA at 56 years of age. Left THA was performed using metal on polyethylene at 57 years of age (Fig. 1). Thirty months after the left THA, she developed pain in the left groin when climbing and descending stairs. Conservative therapy, including rest and trunk muscle training, was performed for approximately 18 months; however, the pain did not improve. There was a distinct difference between left and right hip flexor muscle strength. Physical examination revealed no redness, swelling, or heat around the hip, and the patient did not complain of resting pain. Patrick's test was positive, and tenderness was noted in the Scarpa triangle. The patient also reported pain on dynamic hyperextension of the left hip. The results of blood tests revealed a white blood cell count of $4.06 \times 10^{3} / \mu \mathrm{L}$ and Creactive protein of $0.03 \mathrm{mg} / \mathrm{dL}$. Active infection was not suspected. Culture of the articular fluid was negative. The metal ion concentrations were cobalt $0.5 \mu \mathrm{g} / \mathrm{L}$ and chromium $0.5 \mu \mathrm{g} / \mathrm{L}$. Radiographs showed no clear findings of fracture, osteolysis, or loosening around the joint replace- ment. On plain CT after THA (Fig. 2), protrusion of the metal cup from the edge of the anterior acetabular wall was $10.3 \mathrm{~mm}$. The peri-articular area was poorly assessed due to metal artifact. Imaging using 18F-FDG-PET/CT showed that the iliopsoas muscle was in contact with the protruding cup, and a consistent uptake along the muscle was visible (Fig. 3). The uptake was found inward from the muscle surface facing the anterior edge of the cup. The maximal standardized uptake value (SUVmax) was 5.582 in the early phase and 6.782 in the delayed phase. Uptake within the joint was also noted, suggesting a spillover of inflammation. Though plain MRI showed no evidence of pseudotumor or abnormal changes inside or outside the iliopsoas muscle, similar uptake was noted in the iliopsoas muscle on 18F-FDG-PET/MRI (Fig. 4). However, 18F-FDG-PET/ MRI showed a clearer depiction of the muscle with uptake

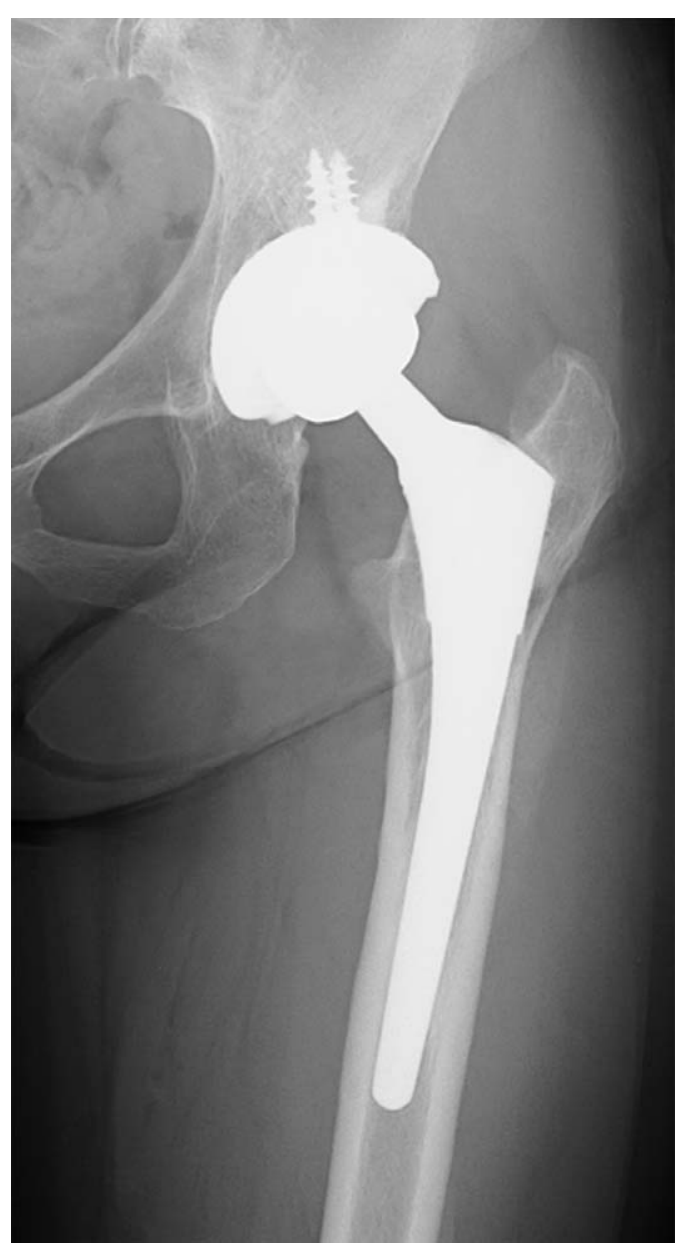

Fig. 1. An anteroposterior pelvic radiograph showing left primary total hip arthroplasty (M2a, a radial acetabular cup of size $50 \mathrm{~mm}$ and a Bi-Metric femoral stem metal ball head with a high-wall polyethylene liner [Biomet, Inc., Warsaw, IN, USA]). 


\section{Hip \& Pelvis}

Makoto Kimura et al. Effectiveness of 118F-FDG-PET/CT and MRI to Detect Iliopsoas Tendonitis after THA

compared to that of 18F-FDG-PET/CT. Based on the progress and image findings after primary THA, we preoperatively considered that the anterior edge of the cup protruding beyond the bone was the main cause of iliopsoas tendonitis. While both open and arthroscopic iliopsoas release were considered for iliopsoas impingement, we selected the isolated acetabular cup revision. This approach was the best option considering the high risk of hindering her ability to perform agricultural work due to hip flexion weakness after iliopsoas release.

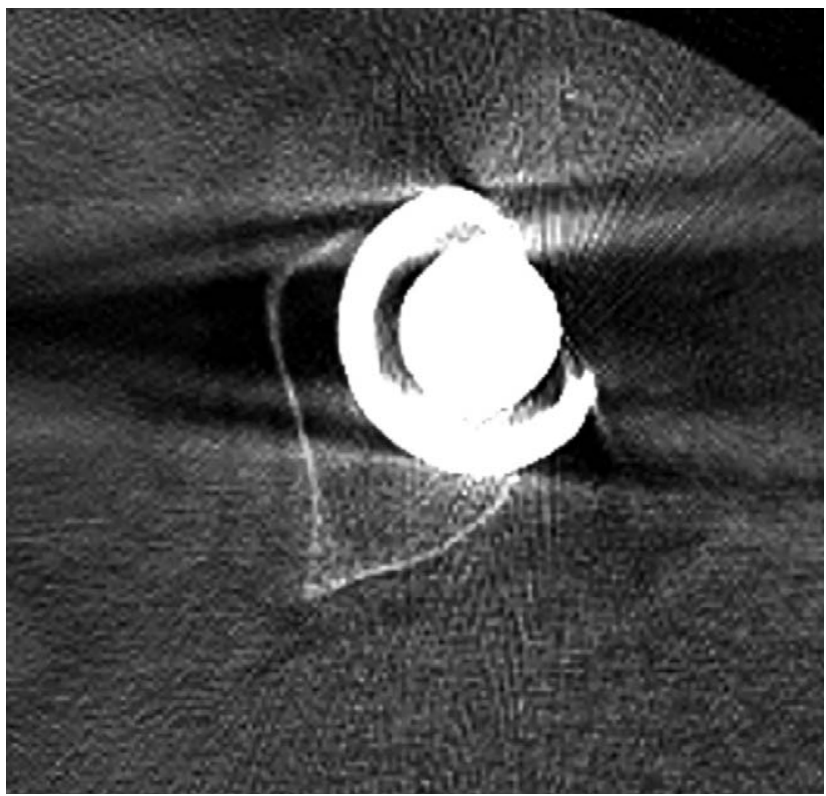

Fig. 2. Plain preoperative computed tomography (CT) image. The cup inclination was $37^{\circ}$ and anteversion was $26^{\circ}$ with stem anteversion of $18^{\circ}$. The front edge of the cup is seen protruding forward on reduction of the CT artifact.
We used the posterior approach for revision. Intraoperative findings (Fig. 5A, B) showed an accumulation of slightly bloody joint fluid in the joint capsule. There was no grossly obvious hematoma or fluid retention in the iliopsoas muscle. No gross evidence of corrosion or wear was noted in the extracted implants (Fig. 5C-E). Revision with retention of the femoral stem was performed under the guidance of a CT-based navigation system (Brain Lab, Munich, Germany) using a smooth-edged cup with a 48-mm diameter (Continuum Acetabular System, Zimmer, Warsaw, IN, USA). The cup was replaced at a more medial position, and the absence of any anterior protrusion that could cause impingement was confirmed intraoperatively (Fig. 6). In the early postoperative period, the patient's pain and ability to perform daily activities improved markedly, including her ability to ascend and descend stairs. The Harris hip score, which was 56 points preoperatively, was 91 points at 6 months postoperatively. The patient was able to achieve the standard clinical outcome that is expected with primary THA.

Per 18F-FDG-PET protocol, the patient fasted for at least 6 hours before examination, and the dose of 18FFDG used was $3.7 \mathrm{MBq} / \mathrm{kg}$. Images using 18F-FDG-PET were acquired from the orbit to the thigh using a PET scanner (BIOGRAPH 40; SIEMENS, Munich, Germany). The patient's blood glucose level was monitored, and the procedure was carried out after confirmation that the level was below $150 \mathrm{mg} / \mathrm{dL}$ prior to injection. The early and delayed phases were performed after 60 minutes and between 90 and 120 minutes, respectively. The PET data were reconstructed in a $168 \times 168$ matrix.

CT was performed using a BIOGRAPH 40 TruePoint
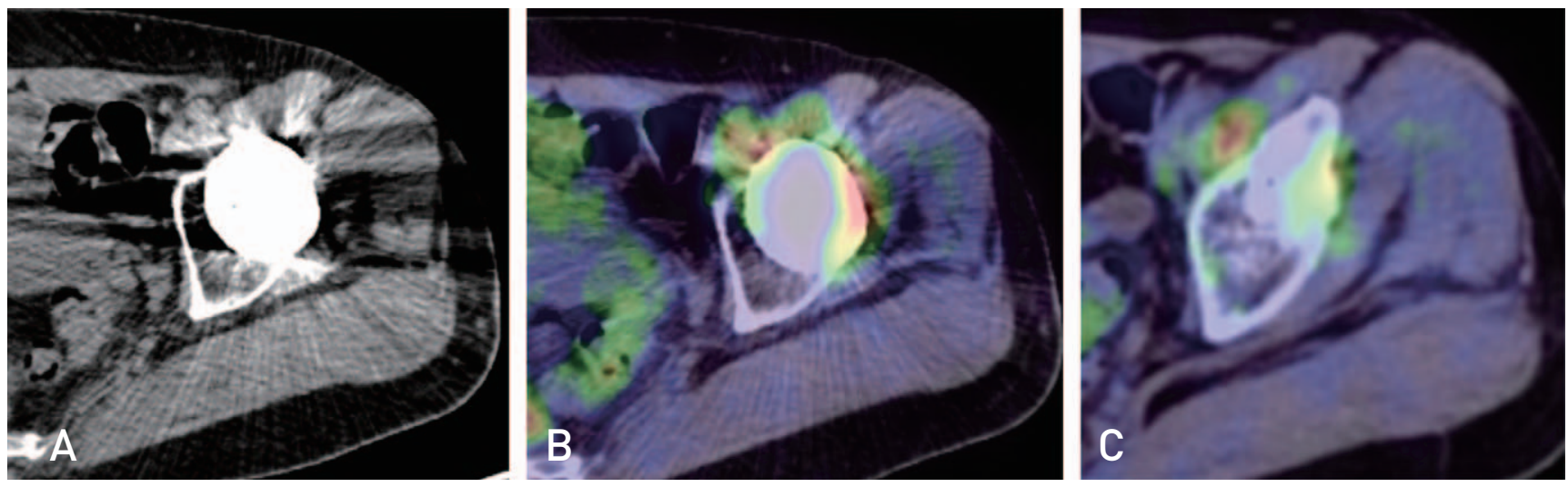

Fig. 3. Plain computed tomography (CT) and fluoro-deoxyglucose positron emission tomography (FDG-PET)/CT images at the same level. (A) Plain CT image with extensive artifact. The soft tissues near and around the prosthesis are difficult to assess. (B) Accumulation seen in the iliopsoas muscle. (C) The FDG-PET/CT image at a slightly higher position than B, and continuous uptake is observed within the iliopsoas muscle on the side of the cup. 


\section{Hip \& Pelvis}

Hip Pelvis 32(4): 223-229, 2020
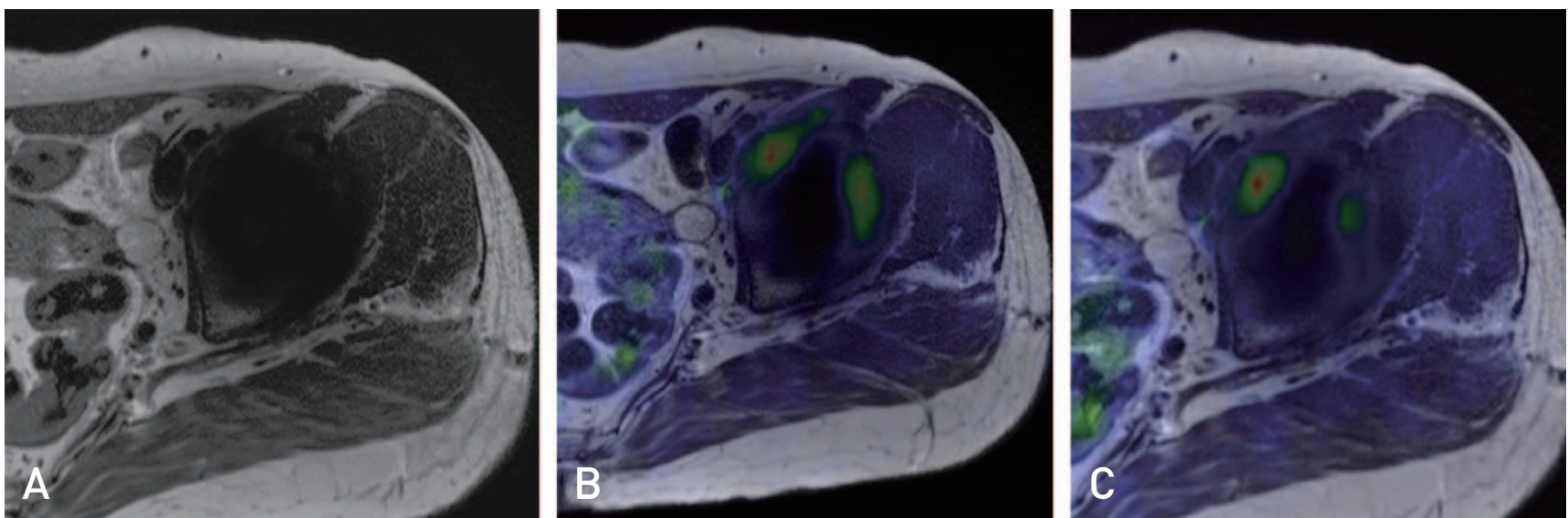

Fig. 4. Plain magnetic resonance imaging (MRI) and fluoro-deoxyglucose positron emission tomography (FDG-PET)/MRI images at the same level. (A) Plain MRI image with extensive artifact complicating assessment of the soft tissues near and around the prosthesis. (B) Accumulation localized at the psoas muscle. (C) The FDG-PET/MRI image at a slightly higher position than $\mathrm{B}$, and continuous accumulation is observed within the iliopsoas muscle on the cup side.
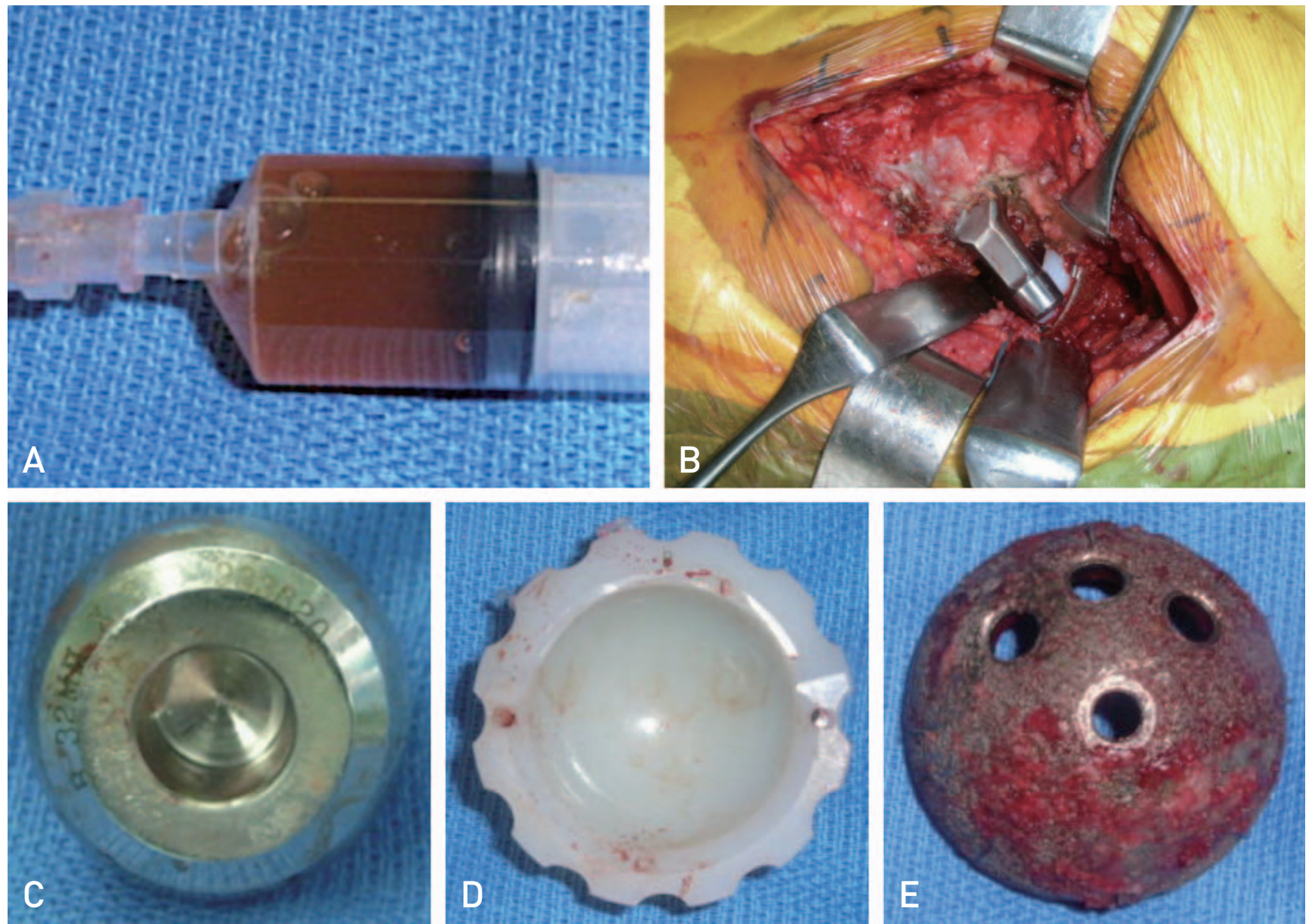

Fig. 5. Surgical findings. (A) Serosanguineous joint fluid with no purulence. (B) No metallosis or necrotic tissue can be seen within the joint. (C) There are no visible signs of metallic corrosion of the head and neck. (D) There is no evidence of excessive polyethylene wear. (E) There is satisfactory osseointegration of the prosthesis with the bone (bone ingrowth). 


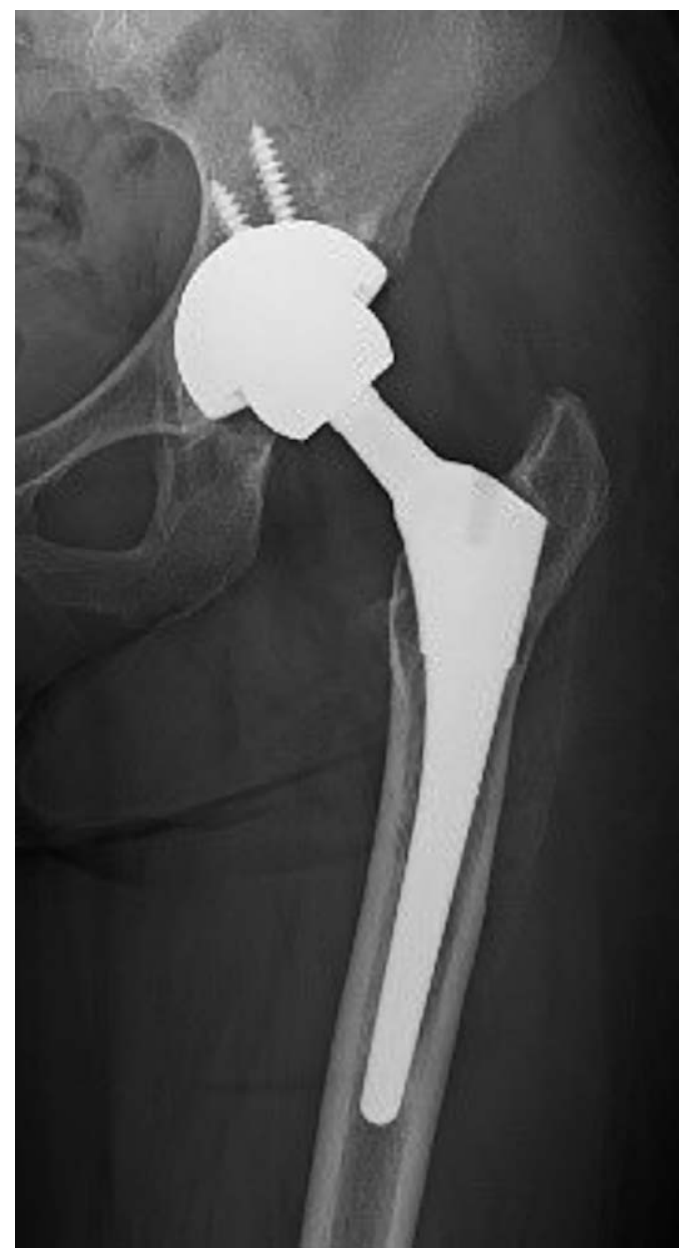

Fig. 6. An anteroposterior pelvic radiograph following left revision total hip arthroplasty. A small-sized cup $(48 \mathrm{~mm})$ was installed deep and internalized with an inclination of $39^{\circ}$ and anteversion of $24^{\circ}$ under the control of the navigation system. The offset polyethylene liner of $7 \mathrm{~mm}$ (Longevity offset liner; Zimmer, Warsaw, IN, USA) with a 6-mm longer ball head of 32-mm diameter was used during the revision surgery to obtain the accurate leg length correction and soft tissue tension for dislocation prevention.

(SIEMENS) with a slice thickness of $3 \mathrm{~mm}$ and a recon spacing of $2 \mathrm{~mm}$. PET was performed using the same parameters and imaging conditions as those of CT $(120 \mathrm{kV}$ and 220 mAs [for CARE Dose4D]).

MRI was performed on a 3T system (Achieva; PHILIPS, Amsterdam, Netherlands) using a 32-channel cardiac coil. The MRIs were obtained as T1-weighted, T2-weighted, and short $\mathrm{T} 1$ inversion recovery images in the coronal and axial planes. The diffusion image was also configured. The metal artifact reduction sequence-MRI (MARS-MRI) was used.

\section{DISCUSSION}

In the current case, a patient presented with pain of unknown etiology after THA. Lesions that could not be visualized by plain CT or MRI due to metal artifacts were detected using 18F-FDG-PET/CT and 18F-FDG-PET/MRI. The lesion was continuously present on the side of the cup within the iliopsoas muscle. Though it was difficult to identify an impingement between the iliopsoas muscle and metal cup dynamically, revision was performed for the diagnosis of iliopsoas tendonitis which was caused by the cup protruding beyond the anterior edge. There were no intraoperative findings to suggest other diseases. After surgery, the patient reported that her pain was resolved.

Anterior groin pain or weakness of hip flexion with iliopsoas tendonitis due to impingement with the cup should be considered a complication after THA $^{9,10}$. Ultrasonography is non-invasive, inexpensive, and useful in diagnosing iliopsoas tendonitis due to iliopsoas impingement after THA as it has real-time imaging functionality during motion ${ }^{11}$. However, the success of ultrasonography depends on the examiner's expertise and may produce an image that is not clear as it does not have equal sensitivity and specificity in all facilities. Alternatively, imaging of iliopsoas tendonitis and inflammatory changes within the muscle located away from the metal can be depicted in plain CT or MRI ${ }^{12}$. Qualitative assessments of areas near implants with metal artifacts remain challenging.

Imaging using 18F-FDG-PET, typically used for the evaluation of neoplastic diseases ${ }^{13}$, has been used to identify inflammatory diseases such as infections and for imaging evaluation after THA. Activation of the glycolytic system by altered glucose metabolism in tumor cells can be assessed by the FDG uptake. An increased FDG uptake in activated inflammatory cells, such as lymphocytes and macrophages, also reflects increased glucose transporter proteins on the cell surface and increased glycolytic metabolism. As a result, such a molecular histological reaction as mentioned above can be visualized as a contrast uptake as in other inflammatory diseases associated with increased glucose metabolism $^{14,15}$. The uptake of FDG in inflammation and other diseases is characterized by a trend toward a decreasing value rather than a marked increase or decrease in dual-time point imaging applications $\mathrm{s}^{16}$. This approach has been used as a tool for objective interpretation of the severity of inflammatory diseases such as rheumatoid arthritis ${ }^{17}$. The evaluation of pseudotumors after metal-on-metal-bearing THA was similarly evaluated using 18F-FDG-PET/CT and has been 
reported to be potentially useful for diagnosis ${ }^{18}$. Reinartz et al. ${ }^{19)}$ found that the PET collection area and uptake values revealed a trend toward infection and loosening of the prosthesis. However, there is no clear diagnostic cutoff value and statistics on SUV values during infection and prosthesis loosening are not available. Choe et al. ${ }^{20)}$ found that the mean SUVs for an infection group and a control group were 11 (5-17) and 4 (3-6), respectively. Reinartz et al. ${ }^{19)}$ also reported a high possibility of infection when the SUV value was over 5-6. Following THA, nonspecific uptake up to SUV 2.5 in contact with the bearing area or the head-neck junction ${ }^{21}$, and intra-articular uptake in contact with the artificial joints should be diagnosed carefully and accurately. Unlike sites with non-specific uptake, a continuous high uptake within the iliopsoas muscle, as in the present case, is a valuable imaging finding that suggests the presence of iliopsoas tendonitis. Herein, iliopsoas tendonitis occurring in the vicinity of an artificial joint was difficult to diagnose by plain CT or MRI due to metal artifacts, but visualization was possible when combined with 18F-FDG-PET. Given that 18F-FDG-PET/CT and 18FFDG-PET/MRI are expensive tools and are still poorly covered by medical insurance in many countries, they should be used with caution in clinical practice. It is advisable to perform PET only when the diagnosis of iliopsoas tendonitis is uncertain following standard ultrasound, plain $\mathrm{CT}$, and MRI examination along with the progression and detailed physical findings in patients. Based on the findings of the current case, CT or MRI used simultaneously with PET enabled anatomical identification of soft tissues, which was useful for the diagnosis of iliopsoas tendonitis. As there was no considerable difference between $18 \mathrm{~F}$ FDG-PET/CT and 18F-FDG-PET/MRI it is not necessary to perform both to identify a lesion in the metal artifact area.

In conclusion, the current case study reports the use of 18F-FDG-PET/CT and 18F-FDG-PET/MRI for the diagnosis of iliopsoas tendonitis after primary THA. Though iliopsoas tendonitis was suspected based on clinical findings and other factors, uptake in the iliopsoas muscle in the vicinity of the joint was noted. Uptake was difficult to visualize on plain CT and MRI due to a metal artifact. We suggest that anatomical identification is possible by overlaying the findings obtained with CT and MRI. Iliopsoas tendonitis after THA should be diagnosed comprehensively considering physical findings and implant placement, along with exclusionary diagnoses. The current study finds that $18 \mathrm{~F}-\mathrm{FDG}-\mathrm{PET} / \mathrm{CT}$ and 18F-FDG-PET/MRI is a useful imaging tool to detect this condition, especially if metal artifacts on plain CT and MRI images are present. As most cases of iliopsoas tendonitis after THA initially occur near the metal implant due to impingement with the cup, use of 18F-FDG-PET/CT and 18F-FDG-PET/MRI are valuable tools for early diagnosis.

\section{CONFLICT OF INTEREST}

The authors declare that there are no potential conflict of interest relevant to this article.

\section{REFERENCES}

1. Forster-Horvath C, Egloff C, Valderrabano V, Nowakowski AM. The painful primary hip replacement - review of the literature. Swiss Med Wkly. 2014;144:w13974.

2. Henderson RA, Lachiewicz PF. Groin pain after replacement of the hip: aetiology, evaluation and treatment. J Bone Joint Surg Br. 2012;94:145-51.

3. Classen T, Zaps D, Landgraeber S, Li X, Jäger M. Assessment and management of chronic pain in patients with stable total hip arthroplasty. Int Orthop. 2013;37:1-7.

4. Adler RS, Buly R, Ambrose R, Sculco T. Diagnostic and therapeutic use of sonography-guided iliopsoas peritendinous injections. AJR Am J Roentgenol. 2005;185:940-3.

5. Dora C, Houweling M, Koch P, Sierra RJ. Iliopsoas impingement after total hip replacement: the results of non-operative management, tenotomy or acetabular revision. J Bone Joint Surg Br. 2007;89:1031-5.

6. Chalmers BP, Sculco PK, Sierra RJ, Trousdale RT, Berry DJ. Iliopsoas impingement after primary total hip arthroplasty: operative and nonoperative treatment outcomes. J Bone Joint Surg Am. 2017;99:557-64.

7. Odri GA, Padiolleau GB, Gouin FT. Oversized cups as a major risk factor of postoperative pain after total hip arthroplasty. J Arthroplasty. 2014;29:753-6.

8. Verberne SJ, Raijmakers PG, Temmerman OP. The accuracy of imaging techniques in the assessment of periprosthetic hip infection: a systematic review and meta-analysis. J Bone Joint Surg Am. 2016;98:1638-45.

9. Bricteux S, Beguin L, Fessy MH. [Iliopsoas impingement in 12 patients with a total hip arthroplasty]. Rev Chir Orthop Reparatrice Appar Mot. 2001;87:820-5. French.

10. Batailler C, Bonin N, M Wettstein, et al. Outcomes of cup revision for ilio-psoas impingement after total hip arthroplasty: retrospective study of 46 patients. Orthop Traumatol Surg Res. 2017;103:1147-53.

11. Jacobson JA, Khoury V, Brandon CJ. Ultrasound of the groin: techniques, pathology, and pitfalls. AJR Am J Roentgenol. 2015;205:513-23.

12. Cyteval C, Sarrabère MP, Cottin A, et al. Iliopsoas impingement on the acetabular component: radiologic and computed tomography findings of a rare hip prosthesis complication in eight cases. J Comput Assist Tomogr. 2003;27:183-8.

13. Lee JW, Lee SM. Radiomics in oncological PET/CT: clinical applications. Nucl Med Mol Imaging. 2018;52:170-89. 


\section{Hip \& Pelvis}

Makoto Kimura et al. Effectiveness of 118F-FDG-PET/CT and MRI to Detect Iliopsoas Tendonitis after THA

14. Mochizuki T, Tsukamoto E, Kuge Y, et al. FDG uptake and glucose transporter subtype expressions in experimental tumor and inflammation models. J Nucl Med. 2001;42:1551-5.

15. Kumar R, Basu S, Torigian D, Anand V, Zhuang H, Alavi A. Role of modern imaging techniques for diagnosis of infection in the era of $18 F$-fluorodeoxyglucose positron emission tomography. Clin Microbiol Rev. 2008;21:209-24.

16. Zhuang H, Pourdehnad M, Lambright ES, et al. Dual time point 18F-FDG PET imaging for differentiating malignant from inflammatory processes. J Nucl Med. 2001;42:1412-7.

17. Chaudhari AJ, Ferrero A, Godinez F, et al. High-resolution (18)F-FDG PET/CT for assessing disease activity in rheumatoid and psoriatic arthritis: findings of a prospective pilot study. Br J Radiol. 2016;89:20160138.

18. Makis W, Rush C, Abikhzer G. Necrotic pseudotumor caused by a metal-on-metal total hip prosthesis: imaging characteristics on (18)F-FDG PET/CT and correlative imaging. Skeletal Radiol. 2011;40:773-7.

19. Reinartz P, Mumme T, Hermanns B, et al. Radionuclide imaging of the painful hip arthroplasty: positron-emission tomography versus triple-phase bone scanning. J Bone Joint Surg Br. 2005;87:465-70.

20. Choe H, Inaba Y, Kobayashi N, et al. Use of $18 F$-fluoride PET to determine the appropriate tissue sampling region for improved sensitivity of tissue examinations in cases of suspected periprosthetic infection after total hip arthroplasty. Acta Orthop. 2011; 82:427-32.

21. Aydin A, Yu JQ, Zhuang H, Alavi A. Patterns of $18 F-F D G$ PET images in patients with uncomplicated total hip arthroplasty. Hell J Nucl Med. 2015;18:93-6. 